\title{
Acquisition of rotation-induced taste aversion as a function of drinking-treatment delay
}

\author{
VAHRAM HAROUTUNIAN and DAVID C. RICCIO \\ Kent State University, Kent, Ohio 44242
}

\begin{abstract}
Four groups of rats received rotation treatment $.5,15,30$, or 120 min after consumption of a saccharin solution. Taste aversion to saccharin developed after a single drinking-rotation session in the .5-min delay group and after three trials in the 15- and 30-min delay groups. No aversion to saccharin was found in the 120-min delay group. Rotation failed to produce learning to nongustatory cues in either CER or passive avoidance paradigms. It was concluded that rotation shared common effects with poisoning and irradiation.
\end{abstract}

That the rat is sensitive to rotational stimulation, despite its inability to vomit, was demonstrated several years ago. Relatively slow rotation speeds of $10-15 \mathrm{rpm}$ produced reliable and marked reductions both in spontaneous activity (Eskin \& Riccio, 1966) and in amount of leverpressing for food on several schedules of reinforcement (Riccio \& Thach, 1968). In the rat, as in species which do display emesis when sick, the effects of rotation are attenuated by destruction of vestibular mechanisms (Riccio, Igarashi, \& Eskin, 1967). These findings, which indicate that a response akin to "motion sickness" can be elicited by rotation in rats, suggest that rotation might also be capable of functioning as a noxious stimulus to support learning. Although Riccio and Thach (1966) reported that Pavlovian pairings of buzzer (CS) and rotation (UCS) failed to produce a CER, they obtained suggestive evidence of conditioned suppression employing a discriminative punishment arrangement. Unfortunately, the highly restricted nature of their study precludes any strong conclusions.

More convincing evidence that rotational stimulation is indeed effective within an associative learning context comes from several recent studies employing conditioned taste aversion as the dependent variable. Green and Rachlin (1973) initially demonstrated the acquisition of an aversion to saccharin-sweetened water when rats were exposed to rotation during drinking sessions. While only two rats were studied, the basic finding has since been replicated by Braun and McIntosh (1973) and Hutchison (1973). Presumably, then, the gastrointestinal consequences of rotation are similar to those produced by agents such as poisoning or irradiation.

One of the striking characteristics of illness-induced taste aversions produced by other agents is the

This study was supported in part by NSF Grant GB 41488 to DCR. The authors acknowledge the helpful assistance of Mary Joan Hess in data collection. Dr. Don Gans, Department of Speech Pathology and Audiology, provided invaluable assistance in developing the procedure for vestibular destruction. Reprint requests should be sent to David C. Riccio, Department of Psy chology, Kent State University, Kent, Ohio $\mathbf{4 4 2 4 2 .}$ prolonged CS-UCS delay over which learning can still occur. Accordingly it seemed of considerable interest to determine whether these unusual temporal relationships might also be true for rotation effects. In this connection, acquisition of taste aversion with a 10 -min interstimulus interval has been reported, but controls for nonassociative changes in drinking preference produced by spinning were not included in the study (Hutchison, 1973). In a well-controlled experiment, Braun and McIntosh (1973) demonstrated acquisition of learned taste aversion with a delay condition of 3 min between consummatory activity and rotation. Longer intervals, however, were not investigated. Accordingly, the primary aim of Experiment I was to examine systematically the acquisition of saccharin aversion as a function of the delay between taste activity and rotational stimulation.

\section{EXPERIMENT I}

\section{Method}

Subjects. Forty-three Holtzman male albino rats were used in this experiment. The subjects were 50 days old at the beginning of the study and had previously participated in a passive avoidance experiment where they received footshock and various dosages of Nembutol (sodium pentobarbital). Subjects were divided into four groups of nine and one group of seven. Food was available at all times.

Apparatus. A phonograph turntable served as the rotation device, rotating at $70 \mathrm{rpm}$ when a metal cage containing the subject was mounted on it. Rotation periods were controlled by a Universal timer (Dimco-Grayco, Dayton, Ohio). A .1\% solution of sodium saccharin $(1 \mathrm{~g} /$ liter $)$ was the stimulus solution.

Procedure. The general design of this experiment included two phases. In Phase I, lasting about 1 week, a stable preference for saccharin over water was established in a two-bottle situation. Phase II consisted of 4-day cycles. On the initial day, separate groups of rats were exposed to rotation treatment $.5,15,30$, or 120 min after a single-bottle saccharin drinking session. On the 2nd day, all rats were tested for saccharin vs. water preference. In order to enhance the discriminative features of the situation, water only, with no rotation, was presented on Days 3 and 4 of the cycle. This sequence was repeated to provide a total of five training and testing trials. As a control for the possibility that severe stress per se might induce aversion, another group of rats received deep body cooling after consuming the saccharin 


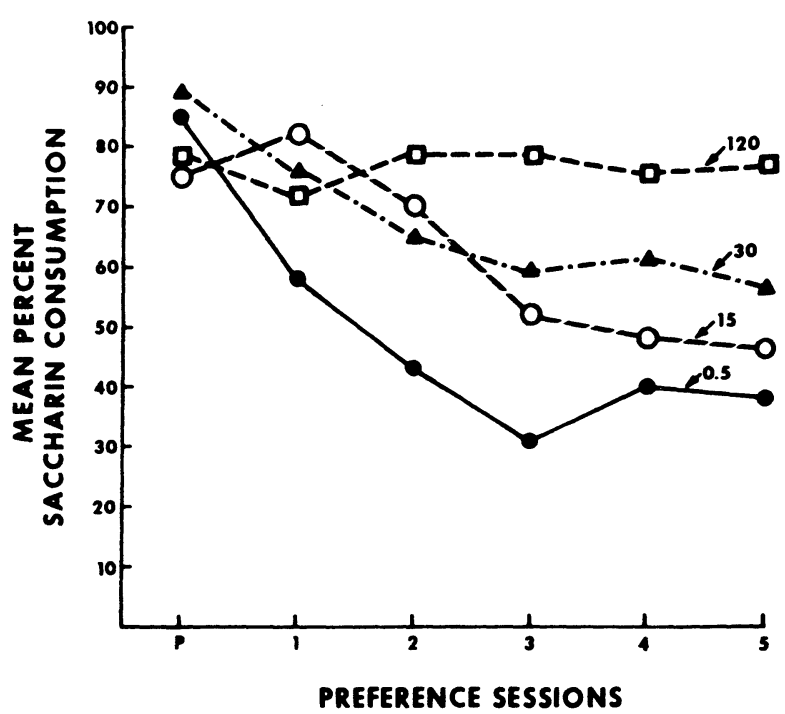

Figure 1. Mean percent saccharin consumption for the four groups on the final baseline preference day $(P)$ and following each of the five taste-rotation sessions. Parameter values refer to the delay (in minutes) between saccharin drinking and onset of rotation.

solution. Since hypothermia produces effects which might be viewed as resembling an internal state of malaise, it appeared that this might be a more appropriate agent than other noxious stimuli such as electric shock or loud noise.

The specific experimental procedures were as follows: At 50 days of age, access to water was gradually decreased to $15 \mathrm{~min} /$ day over a 7 -day period for all subjects. Body weight was reduced to $75 \%-80 \%$ of age mate weight by the end of the adaptation period and maintained there for the duration of the experiment.

Phase $I$. On the days following the adaptation period, subjects were placed individually in large cages $(41.9 \times 24.1 \times 14.6 \mathrm{~cm})$ for $15 \mathrm{~min} /$ day. Bottles of water and saccharin were placed at opposite ends of the cages, position being randomized over days. This procedure was continued until a stable saccharin preference of $70 \%$ of total liquid consumed or better was reached and maintained for 2 consecutive days. This criterion was reached within 5-7 days.

Phase II. Once criterion was reached, subjects received a 15min single-bottle saccharin drinking session in their home cages. At $.5,15,30$, or $120 \mathrm{~min}$ following exposure to saccharin, the rats were exposed to the rotation treatment. In order to reduce the possibility of habituation to rotation, rats received 101 -min rotation sessions at $70 \mathrm{rpm}$. The interrotation intervals were long enough for the cage to come to a complete stop before the beginning of the next rotation $(5 \mathrm{sec})$. Subjects were always rotated in their home cages. On the following day, all animals were tested for saccharin vs. water preference in the two-bottle situation described for Phase I. On the remaining 2 days of this 4-day cycle, subjects received water only, and after the delay interval they were taken to the experimental room but were not rotated. This 4-day cycle was repeated five times.

Retention test. Rats in the .5 -min delay group were allowed ad-lib access to water following the fifth saccharin-water preference test for 21 days. At that point, they were once again deprived of water gradually over 7 days and had access to water for only $15 \mathrm{~min}$ by the 7 th day. Subjects were then given a single 15-min preference test between the two liquids. In order to control for any possible changes in preference over long retention intervals, irrespective of rotation experience, a control group $(\mathrm{N}=7)$ received the same treatment as the experimental subjects up to the end of Phase I. After a preference was established, these rats were given ad-lib access to water for 21 days. Following this period, the rats were gradually water deprived so that by Day 7 they had 15 min access to water. On the next day, they were tested for a water vs. saccharin preference.

\section{Results}

Figure 1 shows the mean percent of saccharin consumed by various delay groups as a function of test days. The values for Session $P$ represent baseline preference obtained on the final session of Phase I. As the figure suggests, changes in rotation induced saccharin preference developed over training trials $(F=15.3$, $\mathrm{df}=5, \mathrm{p}<.001)$ and varied with treatment conditions $(\mathrm{F}=16.74, \mathrm{df}=3, \mathrm{p}<.001)$. The consistently high level of saccharin intake in the 2-h group, in contrast to the diminishing consumption in the shorter delay groups, was reflected in the significant Trials by Treatment interaction $(\mathrm{F}=2.88, \mathrm{df}=15, \mathrm{p}<.001)$. Further analytic comparisons using Newman-Keuls tests indicated that the saccharin preference in the $.5-\mathrm{min}$ delay group was significantly below all other groups after one CS-UCS exposure; by the third test, the .5-, 15-, and 30-min groups each showed less saccharin preference than the 120-min group; and at the end of 5 days of training, mean percent intake differed significantly between each pair of groups, except for the .5 - and 15 -min conditions.

Following the 30-day retention interval, the mean percent intake in the .5-min delay group was 49.9 , and that of the nonrotated saccharin control group was 75.9. These two values are not reliably different $(t=.8$, $\mathrm{p}>.10)$. However, for the .5 -min delay rats, there was also no significant difference in preference level between the last day of acquisition and the later retention test. This pattern of results, while ambiguous, suggests that some modest amount of retention loss occurred during the 1-month interval.

Four additional rats (120-150 days old) received the same treatment as the experimentals with the exception that deep body cooling was used as the UCS (stress control condition). Immediately following the consumption of saccharin in Phase II, these subjects were placed in a wire mesh restraining cylinder and were immersed to the neck in water $\left(2^{\circ} \mathrm{C}\right)$. Rats remained in the water until the body temperature dropped to $22^{\circ}-25^{\circ} \mathrm{C}$. They were then removed, dried with towels, and returned to their home cages. The training-testing cycle was repeated four times.

The data from the stress control rats provided no evidence that saccharin preference was modified by deep body cooling. Mean percent saccharin preference was 82.8 on the last day of prehypothermia preference and 75.3 on Day 5 of posthypothermia treatments. Since these animals were not run concurrently with the main experiment, they were not included in the overall analysis. It is clear, however, that the scores are very 
similar to the 120-min delay condition; moreover, a repeated measures $t$ test indicated no difference between baseline and final saccharin preference $(\mathrm{t}=1.75$, $\mathrm{p}>.05)$.

\section{EXPERIMENT II}

Experiment I clearly demonstrated the acquisition of taste aversion based upon rotational stimulation. Evidence from several other studies indicates that internal malaise may be readily associated with gustatory signals but not with exteroceptive cues (e.g., Garcia \& Koelling, 1966). Conversely, conditioned responses based upon painful stimulation appear to be learned readily to exteroceptive cues but not to taste stimuli (Garcia \& Koelling, 1966). These findings have challenged the notion of equipotentiality of stimuli in conditioning and led some investigators to suggest a dimension of "preparedness" for associating different types of events (Garcia, Hankins, \& Rusiniak, 1974). While a sharp dichotomy between stimulus classes is tempered by recent data showing that rats can learn to avoid a spatial location associated with poisoning (Best, Best, \& Mickley, 1973; Rozin, 1969), it seemed of interest to determine whether nongustatory stimuli could also acquire aversive properties through association with rotation. In Experiment II, we attempted to assess this possibility by pairing distinctive apparatus and contextual cues with rotation and subsequently measuring the degree of suppression of water drinking in the presence of these cues.

\section{Method}

Nine 120-150-day-old rats were water deprived to $90 \%$ of age mate weight over 7 days and were maintained at this weight for the duration of the experiment. The animals had access to water for $4 \mathrm{~h}$ each day. On Days 8 and 9 , subjects were placed inside a box $(19 \times 19 \times 14.6 \mathrm{~cm})$ which was on the turntable. The interior of the box was lined with white cardboard inserts. Subjects remained in this box for $15 \mathrm{~min}$ daily with water available from a graduated Richter tube. On both days, time to onset of drinking, milliliters of water consumed per minute, and total milliliters of water consumed were measured.

Following this 2-day baseline drinking measure, five of the rats received 5 days of $15-\mathrm{min}$ daily exposure to rotation in a black-lined box. A 4,000-cycle tone was paired with the rotation. No water was available during the rotation sessions. Two hours later, these animals were returned to the experimental room and remained confined in their home cage for $15 \mathrm{~min}$.

The four remaining subjects provided a control for noncontingent rotation effects. These rats were not rotated but were confined in the black box for $15 \mathrm{~min}$ and were exposed to the 4,000-cycle tone. Two hours later, these subjects were returned to the experimental room where they received rotation treatments in their home cages. No tones were presented to these subjects during the rotation sessions. Access to water remained restricted to $4 \mathrm{~h} /$ day throughout this tone-rotation phase for both experimental and control subjects. All subjects received water in their home cages $2 \mathrm{~h}$ after the final treatment of each day.

Testing occurred for all animals following the 5-day training period. The subjects were placed inside the black box but were not rotated. The 4,000-cycle tone was present throughout the test session. Subjects were given access to water in this box, and the previously mentioned drinking measures were recorded.

\section{Results}

No evidence of conditioned suppression was obtained following pairing of contextual and auditory cues with rotation. The mean latency to onset of drinking was $125 \mathrm{sec}$ for experimental and $86 \mathrm{sec}$ for controls. While numerically different, these values did not approach significance $(t=1.37, p>.10)$. Similarly, the mean amount of water consumption during the test session was $6.5 \mathrm{ml}$ and $6.6 \mathrm{ml}$ for experimental and control groups, respectively $(\mathrm{t}=.20, \mathrm{p}>.10)$. No differences could be seen in the rate of drinking during the 15 -min test session.

One puzzling outcome was that both groups showed a change in drinking activity between their baseline and test assessments. When data were collapsed across groups, latency to begin drinking was significantly higher $(\mathrm{t}=5.2, \mathrm{p}<.001)$ and total water consumed significantly lower $(t=3.2, \mathrm{p}<.05)$ at the end of the 5 -day treatment period. Since these outcomes were nondifferential with respect to experimental and control conditions, however, they cannot readily be interpreted as evidence of associative conditioning.

\section{EXPERIMENT III}

In contrast to Experiment I, the second experiment failed to provide any substantial evidence of conditioning when exteroceptive cues were paired with rotation. As in any study with negative outcomes, the choice of parameters and sensitivity of the measure employed can be questioned. While we attempted to parallel the procedures of Experiment I, except for the type of $\mathrm{CS}$, it seemed prudent to determine whether conditioning to nongustatory stimuli might be established within a different, presumably more sensitive, paradigm. Toward this end, we employed passive avoidance training. In this type of punishment procedure, the aversive agent is delivered contingent upon the occurrence of a "spontaneous" locomotor response (e.g., crossing from one compartment into another). In testing, the subject is placed on the safe side, and the latency to return to the punished area is measured. Both Pavlovian conditioning to the distinctive contextual stimuli and instrumental punishment of the emitted response appear to contribute to the avoidance performance (Randall \& Riccio, 1969). With electric shock as the punishment, passive avoidance learning is commonly achieved in a single trial. Whether or not rotation is as effective as shock, this procedure would seem to maximize the chances of obtaining learning to nongustatory cues.

\section{Method}

The experimental chamber consisted of two boxes, one white 
and one black, which could be joined together so that a rat could easily cross from one box to the other. The black box, equipped with a guillotine door, was mounted on the phonograph turntable.

Five experimental rats (120-150 days old) were placed individually inside the white box, and the door separating the two boxes was raised. Once the rat entered the black box, the door was lowered, and its cross-through latency was recorded. The white box was then detached, and the black box, containing the animal, was rotated. In order to make the procedure comparable to the procedure used for controls, these subjects were returned to the experimental room $2 \mathrm{~h}$ following rotation and remained there confined in their home cages for $15 \mathrm{~min}$.

To control for any possible effects of rotation alone, a noncontingent control group (five 120-150-day-old rats) received the same treatment as the experimentals with the exception that on crossing through to the black side, they received no rotations but were confined there for $15 \mathrm{~min}$. Two hours later, they were returned to the experimental room and were rotated in their home cages.

These procedures were repeated once a day for 8 days for both groups. On Day 9 , in addition to measuring cross-through latency, time spent in the black side was determined for a 5-min period. Subjects were not rotated on this day and had access to both compartments.

\section{Results}

No differences were found in the latency to step through into the black side of the apparatus between the experimental and control groups even after nine experimental trials. Mean step-through latencies were $3.5 \mathrm{sec}$ for the experimental group and $3.2 \mathrm{sec}$ for the control $(\mathrm{t}=.54, \mathrm{p}>.10)$. Moreover, both groups showed slightly shorter latencies across sessions, a trend directly opposite to what would be expected with' acquisition of a passive avoidance response. (Mean initial latencies were 5.4 and $5.8 \mathrm{sec}$ for experimental and control subjects, respectively.) Further evidence that the area associated with rotation did not become aversive is seen in the fact that the experimental group spent most of its time in the black compartment (264 out of $300 \mathrm{sec})$. This value did not differ from that of controls $(254 \mathrm{sec})$.

\section{GENERAL DISCUSSION}

These findings show that taste aversion based on rotation can be established despite prolonged intervals between the critical stimulus events. While clear evidence for suppression of saccharin drinking was found in the 30-min delay condition, the degree of learned aversion increased as the temporal delay between saccharin consumption and rotation decreased. Acquisition of the aversion was relatively rapid, occurring after a single treatment in the shortest delay condition and after three treatments for the 15- and 30-min conditions. These changes cannot readily be attributed to neophobic reactions or general changes in saccharin preference from rotation per se since the 2 -h delay group showed no significant shift in saccharin preference. Retention of the learned aversion, while not parametrically studied, appeared to be reasonably substantial in the .5 -min delay group even 1 month after the last rotation exposure.

These outcomes suggest that, in terms of the learning consequences, the malaise produced by rotation shares considerable commonality with that elicited by potentially more lethal agents such as $\mathrm{LiCl}$ or irradiation. The conditioned taste aversion induced by rotation appears to be related specifically to internal malaise rather than systemic stress. Inducing deep body cooling shortly after tasting saccharin had no observable influence on taste performance. 1 Similarly, Braun and McIntosh (1973) have reported that prolonged intense noise as a stressor was ineffective in producing taste aversion.

Casual observation and anecdotal reports have indicated that children who become "car sick" may tend to resist going for rides but not necessarily develop aversions to their most recent meal. It seemed possible that the associability of motion-induced sickness might lie somewhere between that of poisoning illness (gustatory) and footshock (exteroceptive). Accordingly, we expected rotation to show some characteristics of both classes of aversive agents and to support at least partial learning to both gustatory and nongustatory stimuli. The second major finding here is that learned avoidance based on rotation occurs much more readily, and perhaps exclusively, to gustatory cues rather than proprioceptive or exteroceptive stimuli. At the very least, rotational experience which was sufficient to produce marked taste aversion did not yield evidence of response suppression in either CER or passive avoidance paradigms. Although it is possible that some aversion to the black box did occur in the passive avoidance paradigm, this aversion could not have been of sufficient strength to overcome the subject's preference for the black box. These findings appear consistent with and supportive of interpretation by Garcia et al. (1974) and Seligman (1970).

Finally, it is generally assumed that the vestibular system is importantly involved in mediating malaise during rotation. While there is evidence that even partial vestibular destruction markedly attenuates the behavioral disruption ordinarily produced by rotation in rats (Riccio et al., 1967), direct evidence of the role of labyrinth mechanisms in rotation-induced taste aversion has not previously been presented. In this connection, subsequent to the main experiment reported above, six rats were subjected to gross bilateral destruction of the vestibular area using an electrolytic procedure (Haroutunian, Riccio, \& Gans, Note 1). Following a recovery period of several weeks, these rats were subjected to the same treatment as the .5-min delay group of Experiment I. No suppression of saccharin drinking was found in these animals. (Mean percent consumption was 84.6 and 77.6 for the last 2 days of baseline and after five rotation trials, respectively.) Thus, the taste aversion appears to be linked importantly with vestibular stimulation, rather than other concomitants of rotation. 


\section{REFERENCE NOTE}

1. Haroutunian, Riccio, \& Gans, 1975. In preparation.

\section{REFERENCES}

Best, P. J., Best, M. R., \& Mickley, G. A. Conditioned aversion to distinct environmental stimuli resulting from gastrointestinal distress. Journal of Comparative and Phy siological Psychology, 1973, 85, 250-257.

Braun, J. J., \& McIntosh, H. Learned taste aversions induced by rotational stimulation. Physiological Psychology, 1973, 1 , $301-304$.

Eskin, A., \& Riccio, D. C. The effects of vestibular stimulation on spontaneous activity in the rat. Psychological Record, $1966,16,523-527$.

Garcia, J., \& Koelling, R. A. Relation of cue to consequence in avoidance learning. Psy chonomic Science, 1966, 4, 123-124

Garcia, J., \& Hankins, W. G., \& Rusiniak, K. W. Behavioral regulation of the milieu interne in man and rat. Science, 1974, $185,824-831$.

Green, L., \& Rachlin, H. The effect of rotation on the learning of taste aversions. Bulletin of the Psychonomic Society, 1973, 1, 137-138.

Hutchison, S. L. Taste aversion in albino rats using centrifugal spin as an unconditioned stimulus. Psychological Reports, $1973,33,467-470$.

Krane, R, V., \& Wagner, A. R. Taste aversion learning with a delayed shock UCS: Implications for the "generality of the laws of learning." Journal of Comparative and Physiological Psy chology, 1975, 88, 882-889.

Randall, $P$. K \& Riccio, D. C. Fear and punishment as determinants of passive avoidance responding. Journal of Comparative and Physiological Psychology, 1969, 69, 550-553.
Riccio, D. C., Igarashi, M., \& Eskin, A. Modification of vestibular sensitivity in the rat. Annals of Otology, Rhinology, Laryngology, 1966, 76, 179-189.

Riccio, D. C., \& Thach, J. S. Response suppression produced by vestibular stimulation in the rat. Journal of the Experimental Analysis of Behavior, 1968, 11, 479-488.

Riccio, D. C., \& Thach, J. S. Rotation as an aversive stimulus for rats. Psy chonomic Scie nce, 1966, 5, 267-268.

Rozin, P. Central or peripheral mediation of learning with long CS-UCS intervals in the feeding system. Journal of Comparative and Phy siological Psychology, 1969, 67, 421-429.

Seligman, M. F. P. On the generality of the laws of learning. Psy chological Review, 1970, 77, 406-418.

\section{NOTE}

1. Krane and Wagner (1975) have recently presented an intriguing reinterpretation of taste aversion learning. Since the duration of the UCS is suggested as one of the critical variables, employment of rotation or hypothermia may prove useful in evaluating the model. While the debilitating effects of hy pothermia persist for several hours, preliminary data from our laboratory suggest that the duration of the rotation induced sickness is quite brief, perhaps on the order of minutes.

(Received for publication January 13, 1975; revision accepted March 18, 1975.) 